\title{
Mobile apps for orthopedic surgeons: how useful are they?
}

This article was published in the following Dove Press journal:

Orthopedic Research and Reviews

14 May 2015

Number of times this article has been viewed

\section{Orrin I Franko' \\ John P Andrawis ${ }^{2}$ \\ Dayne T Mickelson ${ }^{3}$}

'Department of Orthopedic Surgery, University of California, San Diego

Medical Center, San Diego, CA, USA; ${ }^{2}$ Department of Orthopedic Surgery, Harbor-University of California, Los Angeles Medical Center, Torrance, CA, USA; ${ }^{3}$ Department of Orthopedic Surgery and Sports Medicine, University of Washington, Seattle, WA, USA
Correspondence: Orrin Franko Department of Orthopedic Surgery, University of California, San Diego, 9500 Gilman Drive, La Jolla, CA 92093, USA.

Email orrin@toporthoapps.com
Abstract: Smartphone apps have become an integral part of medical training and practice in the hospital and clinical setting. However, to ensure the safety of patient care, it remains imperative that physicians and physician-educators alike continue to monitor and recognize the strengths and limitations of these powerful tools. Orthopaedic surgeons have widely adopted the use of smartphones and tablets and consequently, a number of resources have emerged to assist orthopaedic trainees and providers in discovering and assessing the most appropriate apps for their practice. The purpose of this review article is to advise readers on how best to identify apps for orthopaedic surgeons, summarize the most popular and useful existing apps, present the current available data to support their use, and provide recommendations to the orthopaedic community regarding safe and responsible mobile technology use in clinical practice.

Keywords: smartphone, tablet, mobile, apps

\section{Introduction}

The rapid and widespread adoption of smartphones and tablets among physicians has resulted in an explosion of creative uses of these mobile devices and their associated software applications (apps). While early research and literature evaluating orthopedic mobile apps is over-represented, ${ }^{1}$ the prevalence of smartphones in medicine is now ubiquitous among all specialties, and research on the subject is growing exponentially (Figure 1). Since the majority of medical trainees in the US use smartphone apps in their clinical practice, ${ }^{2}$ it remains imperative that physicians and physician-educators alike continue to monitor and recognize the strengths and limitations of these powerful tools.

Orthopedic surgeons used smartphones as teleconsultation and communication tools years before apps were made available. ${ }^{3,4}$ The first publication specifically addressing mobile apps designed for orthopedic surgeons was published by Franko in $2011 .^{1}$ That study identified a total of 74 apps that would be of interest to orthopedic practitioners and patients. Since that time, a number of other publications have revised and updated the list of current apps available for orthopedics. ${ }^{5,6}$ The estimated total number of orthopedic-focused apps currently available on the two most popular platforms (iOS and Android) is around 250-300, depending on the criteria used to define an app as "orthopedic-focused". The variety of orthopedic mobile apps available spans a large range of categories including patient education, physician education, clinical evaluation, clinical treatment, and surgical training. In addition, medical device developers have recently started to include mobile smartphone integration with their existing implants and surgical tools. This combination of medical devices with smartphone apps is still 


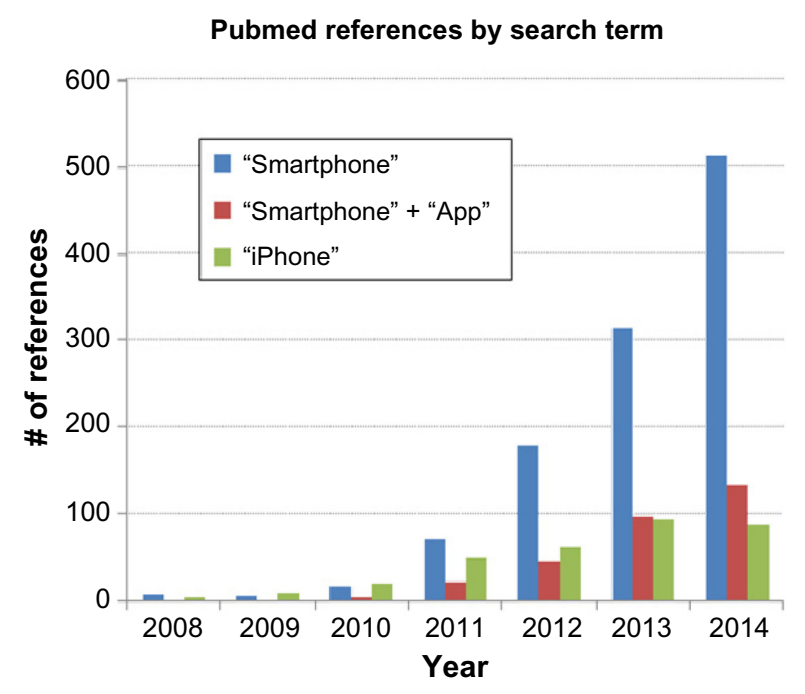

Figure I Number of Pubmed references for smartphone and app-related search terms from 2008 to 2014 .

in its infancy, and this paper will focus on the existing and widespread apps that are currently available.

The March 2014, the Mobile Technology and Social Media Usage study from the Journal of Bone and Joint Surgery (JBJS) reported that, on average, orthopedic surgeons spend about 42 minutes a day on tablets. ${ }^{7}$ Similarly, according to the JBJS 2014 Readership study, $76 \%$ of residents use medical apps on their phones, and it is expected that over the next 2 years, apps will play an even more important role in daily activities. ${ }^{8}$ This study also reported that over half of orthopedic residents predict that they will rely heavily on mobile apps to obtain clinical orthopedic information in the future. In contrast, practicing orthopedic surgeons did not share the same reliance, with only $36 \%$ reporting that they are likely to rely heavily on mobile apps, instead preferring online and print journals as references. ${ }^{8}$ As the next generation of technology-oriented surgeons progress through training and begin practicing, it appears inevitable that app popularity and desirability will only grow. However, it is difficult for any individual to stay abreast of the expanding app library due to the rapid changes in software development and publications. Consequently, a number of resources have emerged to assist orthopedic trainees and providers in discovering and assessing the most appropriate apps for their practice.

The purpose of this review article is to 1) guide readers on how to best identify apps for orthopedic surgeons, 2) summarize the most popular and useful existing apps, 3) present the current available data to support their use, and 4) provide recommendations to the orthopedic community regarding safe and responsible mobile technology use in clinical practice. This paper is a static article on a dynamic topic and suffers from inherent limitations. As a result, parts of this article will be outdated by the time of publication. Nevertheless, many of the principles stated here should remain true, and can help guide the safe integration of apps into practice even as the library of orthopedic apps evolves.

\section{Discovery resources}

Finding apps that are relevant and useful can be a challenge and has largely been accomplished by asking colleagues or searching the App Store or Android Market for appropriate key terms. However, published numbers from July 2014 suggest that these app stores offer 1.2 and 1.3 million apps, respectively. ${ }^{9}$ Not surprisingly, this method of independently searching these digital stores commonly generates an inundation of results that are neither reputable nor valuable. Two reports from American EHR Partners supported this conclusion when they surveyed 1,400 physicians and found that only $28 \%$ of smartphone users and $18 \%$ of tablet users were "Very Satisfied" with the quality of apps for their profession, further illustrating the challenge of app discovery for all providers. ${ }^{10,11}$

There are a number of online resources meant to assist health professionals find medical apps. Apple launched a new App Store section in September 2011 called "Apps for Healthcare Professionals" in response to the recognized value of medical apps. The section is found within the "Medical" App Store and is divided into seven categories: Reference, Medical Education, EMR and Patient Monitoring, Nursing, Imaging, Patient Education, and Personal Care apps. While the effort to separate health professional apps is appreciated, browsing the thousands of subcategorized offerings can still be cluttered and nonspecific. In Apple's new iOS 8 platform, an additional "Explore" section was added to the app store to help users sort through the clutter and find apps most applicable to them. While it still has limited search functionality, there is an orthopedic area found within the "Medical" section (subtopic "Surgery", sub-sub topic "Orthopedic"). It is a significant improvement to the previous App Store search functionality, but it is still suboptimal in helping orthopedic surgeons narrow down to those most relevant to their practice.

Another resource created specifically for orthopedic related mobile apps is http://www.TopOrthoApps.com. The website was started after publication of the first orthopedic app review in 2011. ${ }^{1}$ This resource aims to guide those interested in discovering the best apps for their orthopedic practice by methodically evaluating and summarizing the key features of each orthopedic app. http://www.TopOrthoApps.com has reviewed over 200 apps and may be useful to readers looking for apps by specialty, device platform, or cost. 
In addition, there are a number of other websites and blogs focused on reviewing apps for the medical community. For example, England's National Health Service runs its own online Health Apps Library of verified and recommended

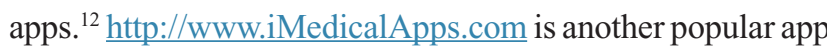
review website that focuses on rating and reviewing apps for all medical specialties. Within the website, they have a section specifically reviewing orthopedic related mobile apps. iMedicalApps.com includes resourceful apps that are not specific to musculoskeletal pathology, but useful for general medical tasks such as medical calculators, drug references, and productivity tools.

No perfect app search utility tool for orthopedic surgeons currently exists. In addition, websites such as those discussed above rely on reviews by a single or a small group of individuals, which have the potential to bias their evaluations and recommendations. Thus, we advise those interested in keeping up with the most recent apps to regularly review the sites listed above, provide their own app reviews within the app stores, ask friends and colleagues for recommendations, and read "technology updates" in orthopedic journals, magazines, and conferences where new products are often announced and reviewed first.

\section{Functionality}

The variety of apps that one will discover when using the aforementioned websites is vast. Currently there are apps intended for orthopedic patients, surgeons, other health care professionals, and industry representatives. Determining the target audience for each app is not always easy, and most apps do not offer a free version to trial before purchasing for download. The purpose of an app may focus on education of disease, education of products, surgical simulations and education, clinical testing and treatment, and much more. One study examined the types of apps that are most useful to orthopedic surgeons and trainees. An online survey of Accreditation Council for Graduate Medical Education (ACGME)-accredited orthopedic training programs was conducted distributed over seven time points between August 2010 and August 2014. ${ }^{13}$ When asked to select the most useful category of apps, the top five responses were Technique Guides (35\%), Coding and Billing (34\%), Textbooks/Reference (32\%), Orthopedic In-Training Exam (OITE)/Board Study Material (28\%), and Classification/ Treatment (27\%). While the peer-review literature does not address many of these topics, some attempts have been made to evaluate and clarify the utility of various apps.

Currently, the most widely "validated" apps include a number of goniometric (angular measurement) tools. For example, published articles have demonstrated the validity of using smartphones to measure acetabular cup orientation, ${ }^{14}$ Cobb angles in scoliosis, ${ }^{15,16}$ shoulder range of motion, ${ }^{17}$ rib hump measurements, ${ }^{18,19}$ knee range of motion, ${ }^{20,21}$ and wrist motion. ${ }^{22}$ All of these studies demonstrate that the accelerometer and gyroscope within smartphones are highly accurate, and thus these apps can provide reliable angular measurements if the device can be appropriately secured to the limb or region being measured. The value of electronic versus standard goniometric measurements remains surgeon-dependent. While there is no question that the measurements are highly accurate and reproducible, the clinical need for such a high degree of precision may not outweigh the inconvenience of using a smartphone for this task. Interested providers should try a free or inexpensive goniometer app to determine if this solution will be effective within their existing work flow (Table 1A).

Another category of validated smartphone apps includes remote consultation or image viewing tools. ${ }^{23,24}$ Once again, these studies demonstrate that viewing images on mobile devices can be highly accurate and increase the efficiency of patient care in remote settings or when an orthopedic surgeon is not immediately available. Image resolution and clarity on mobile devices has been an issue addressed with the increased resolution of tablet and smartphone screens. ${ }^{25}$ Using devices to view medical studies also alludes to the concern over privacy. Thus, it is important to enable a

Table I The "top" orthopedic surgery apps - December 2014

\begin{tabular}{ll}
\hline A: Goniometers & B: Image sharing \\
Goniometer Pro & $\begin{array}{l}\text { DocSpera } \\
\text { PingMD }\end{array}$ \\
Scoligauge & TigerText \\
Simple Goniometer & D: Patient education \\
C: Textbooks/journals & DrawMD Orthopedics \\
JBJS Journals & HandCare \\
Insights Orthopedics & Orca MD \\
AAOS eBooks & Orthopedic Patient Information \\
Campbell's Operative Orthopedics & Skeleton System Pro III \\
& F: Patient resources \\
E: Device companies & Heel Thy Tendon \\
Biomet Bone Model & iPrevent ACL Injuries \\
Sigma Knee Replacement & My Knee Guide \\
Zimmer 4II & PhysioMD \\
& ShoulderDoc \\
& H: Physical therapy/exam \\
G: News/magazine & CARE for Patients \\
AAOS Now BoneFeed & CORE Clinical Orthopedic Exam \\
Medscape & Get Set \\
Orthopedics Today & iOrtho+ \\
& Meniscus Exercises \\
& Throw Like a Pro \\
\hline
\end{tabular}


device's passcode, and there are a number of commercial apps available to assist with encryption, secure messaging, and safe image viewing. If used, secure image-sharing apps and case-sharing platforms can be valuable features of mobile devices (Table 1B).

The number of apps that have been peer-reviewed and validated by the orthopedic community is limited to those listed above, but there exist many more app categories that residents and surgeons alike may find useful. For example, providers regularly rely on journals and textbooks for generalized education as well as subject-specific research and reference. A majority of the most popular orthopedic journals have developed mobile versions of their journals, which include searchable, downloadable, full-text access for institutional or individual subscribers. The most obvious benefit to digital journals is the ability to store thousands of articles on a single device, and many of these journal apps have functionality that allow the user to highlight, share, and save text. In addition, there exist stand-alone portable document format (PDF) readers that have the added benefits of enhanced storage, document management, browsing, synchronization, and annotation features and can serve as a complement to existing journal-sponsored apps. Using mobile apps for literature reading is one of the most useful features of tablets, and a critical tool for any orthopedic surgeon (Table 1C).

Another popular category of useful apps includes patient education resources. Although not validated, a number of anatomy and musculoskeletal pathology apps provide information, images, and videos to help surgeons better educate their patients about specific diseases and treatment options. In many cases, the apps including the ability to draw on images, highlight various parts of the anatomy, and share pictures directly with the patient. Additionally, patients have reacted positively to tablet computers when used to enhance their care. ${ }^{26}$ The best apps in this category are not free, but are still a very good value for the $\$ 20-50$ price range. For a surgeon who likes to draw out pathology for patients who are visual learners, these apps can be very useful during office visits (Table 1D).

The majority of orthopedic device companies have also created apps for surgeons and patients as alike. While some of these apps focus more on patient education, others emphasize product education for the surgeon. These may include surgical technique guides, product guides, implant catalogs, and preoperative templating tools. Since much of this information is widely available and easily accessible on the World Wide Web, these apps are only moderately useful. However, for surgeons who prefer to have this information only a few taps away at all times, these apps may be of benefit (Table 1E).

A number of apps have also been developed by surgeons or their practices as custom educational resources for their patients. These apps tend to focus on a particular pathology, such as treating foot pain or meniscus injuries, and offer suggestions for treatment or guidance to see a surgeon. Some surgeons have even developed sophisticated apps that include preoperative, perioperative, and postoperative information for patients planning to undergo orthopedic procedures (Table 1F).

Mobile devices can be an ideal platform for keeping abreast of the latest orthopedic news and information. Beyond journals and books, there exist a number of orthopedic magazines and medical news platforms that have created mobile apps. Updates occur daily to provide the latest and most relevant information to readers, and some apps even offer Continuing Medical Education (CME) credit (Table 1G).

With increasing strain placed on the health care system as a whole, there has been an effort to offload direct care providers when possible. One shift, accelerated by technology, is the transition to at-home physical therapy through either online or mobile guidance. Not surprisingly, a number of apps have been created that demonstrate physical therapy exercises, stretches, and physical examination tools for therapists and trainees alike. These apps are of very high quality and can be useful to both patients and providers (Table 1H).

A number of companies have specifically targeted orthopedic resident education to improve training and patient care. Some of these apps include an abbreviated and condensed form of existing textbooks, while others utilize the device to provide images, videos, and text to help junior residents effectively navigate a night on call. This article is not intended to provide a detailed description of all available apps for residents, but we include a short list of the most high-yield apps that any resident should find useful (Table 2). Any surgeon looking to expand their smartphone with orthopedic knowledge should start by carefully reviewing Tables 1 and 2 .

Table 2 "Top" resident education apps - December 2014

Resident education
AO Surgery Reference
AO OrthoGeriatrics
AO/OTA Classification
BoneDoc
ICUC
Nerve Whiz
Traumapedia
TouchSurgery

AO Surgery Reference

AO OrthoGeriatrics

BoneDoc

Nerve Whiz

TouchSurgery 


\section{Risks, limitations, and oversight}

As the use of apps and mobile devices within orthopedics grows, it is important to recognize that not all apps are validated or of high quality. Inappropriate use can lead to unintended consequences. ${ }^{27} \mathrm{App}$ developers are not obligated to divulge the sources, guidelines, or limitations of their tools, and some apps require users to accept a legal disclaimer when logging in. It is ultimately the responsibility of the user/physician to decide the credibility and safety of the app. Hence, the potential for mobile apps to negatively impact patient safety is real, and has been shown to affect patients in relation to bacterial contamination, ${ }^{28,29}$ melanoma detection, ${ }^{30}$ opioid conversion, ${ }^{31}$ smoking promotion, and poor guideline adherence. ${ }^{32}$ This has led to recommendations of increased regulation for certain clinical apps. ${ }^{33,34}$ The Food and Drug Administration (FDA) itself does not have the resources to adequately monitor all medical apps, but has indicated that they will provide oversight of apps that meet certain criteria. ${ }^{35}$ Specifically, the FDA will focus primarily on apps that are intended to be used as an accessory to a regulated medical device or transform a mobile platform into a regulated medical device and present a greater risk to patients if they do not work as intended. As a result, the majority of medical apps in the marketplace are essentially unregulated at this time.

The lack of physician involvement in app development and oversight is of continued concern..$^{27,31,36-38}$ It has been proposed that a government-level health care app store be developed; ${ }^{39}$ however, this would likely become burdensome and inefficient. Oversight may be improved if conducted on a professional organizational level (eg, American Academy of Orthopedic Surgeons, AAOS), local health organization level, or individual institution level where hospitals could advocate and support the use of apps among their health care providers.

In addition to lack of oversight for app content, any patient information that is shared on mobile devices is at risk of a security breach. The Health Insurance Portability and Accountability Act (HIPAA) of 1996 was designed to protect the confidentiality of protected health information (PHI). However, the transmission of PHI wirelessly and among mobile devices results in the threat of a HIPAA violation by theft or hacking. As a result, security has become a significant concern for mobile app developers. While HIPAA requires reasonable steps to be taken to ensure confidentiality, there are no specific guidelines for software, and the execution and implementation of security is left to the developer. The Federal Trade Commission recommends that app developers encrypt sensitive data when transmitting information or storing information on a device, ${ }^{40}$ but there is no guarantee this is being done. Privacy Rights Clearinghouse, a nonprofit organization meant to help make consumers aware of how technology affects their personal privacy, examined 43 paid and free health and fitness apps and found that only $13 \%$ of free apps and $10 \%$ of paid apps encrypted all data connections between the app and the developer's website and 39\% of the free apps and $30 \%$ of paid apps sent data to someone not disclosed by the developer or in their private policy. ${ }^{41}$ To avoid privacy issues, developers have often opted to exclude the ability to save or store any patient information within an app. In other cases, users can save PHI that is stored only on an encrypted cloud-based server. Thus, because no patient information is ever stored on the mobile device, the user is protected from a potential breach as a result of device theft. Users who download and plan to use communication tools that require the sharing of PHI should carefully evaluate the security procedures taken by the app developer and ensure that patient information is not at risk.

Although limited oversight and HIPAA violations will always remain a concern, the potential pitfalls from the use of medical apps is likely mitigated by improved education, communication, and knowledge dissemination to patients and health care providers. These risks to the medical community vary in the degree of severity, who is negatively affected, and how they are best managed. ${ }^{42}$ In fact, a simple riskstratification "app-space" framework has been described by Lewis and Wyatt to predict the risk posed by a specific app and promote safe use. ${ }^{42}$ Depending on the app's functionality and risk assessment, the recommended regulation and safety steps vary from a clinician's self-assessment of the app, to peer review of the app, to best practice guidelines, to regulation by a professional or government body. However, until better oversight and safety standards are in place, orthopedic surgeons must take the responsibility for the apps they use for patient care. They should use clinical judgment and work toward verifying validity and adopting current institutional regulations for app integration. In addition, because no specific standards exist to regulate medical devices, developers should work with physicians and take the necessary precautions with regard to encryption and software security to ensure that no potential breach of protected patient information is likely to occur.

\section{Recommendations for integration}

Since the release of the iPhone in 2007, in combination with the ensuing app development boom, there has been a revolution in the way we access and process information. Yet, over 
this time period, there still exists a need for higher quality orthopedic apps. With only 27,773 known practicing orthopedic surgeons and an average age of 54.85 , the market may not currently be very appealing to an app developer. ${ }^{43}$ As a result, many of the best apps within our field have been created by orthopedic associations or groups with a limited budget and limited software development skills. On the other hand, apps released by dedicated developers and major corporations demonstrate a palpable difference in the quality, and often the utility, of the final product. As such, we believe there exists an opportunity for surgeons to partner with organizations to create and improve the orthopedic app market, especially as the young tech-savvy surgeons grow within the specialty.

As technology advances, companies are recognizing that the impact smartphones and their apps will have on physicians and patients alike will continue to evolve. In their new iOS 8 platform, Apple has created its own Health app that will aggregate health information from a range of other iOS apps, wearable devices, and Bluetooth equipped monitors. While this may not directly affect an orthopedic surgeon's practice, more patients will become familiar with using mobile devices to generate, report, and ascertain health information. As this trend continues, orthopedic surgeons have a great opportunity to leverage this shift and improve patient care, while streamlining their practice through the use of mobile technology and apps.

\section{Disclosure}

Dr. Franko is the owner of www.TopOrthoApps.com, as well as consulting affiliations related to app development for AO Foundation, Insights Orthopedics, Computer Aided Rehabilitation Education, DocSpera, ResQ Medical, Lineage Medical, and NewportMed. Dr. Andrawis and Dr. Mickelson are both editors for www.TopOrthoApps.com The authors report no other conflicts of interest in this work.

\section{References}

1. Franko OI. Smartphone apps for orthopaedic surgeons. Clin Orthop Relat Res. 2011;469(7):2042-2048.

2. Franko OI, Tirrell TF. Smartphone app use among medical providers in ACGME training programs. J Med Syst. 2012;36(5):3135-3139.

3. Ortega GR, Taksali S, Smart R, Baumgaertner MR. Direct cellular vs indirect pager communication during orthopaedic surgical procedures: a prospective study. Technol Health Care. 2009;17(2):149-157.

4. Chandhanayingyong C, Tangtrakulwanich B, Kiriratnikom T. Teleconsultation for emergency orthopaedic patients using the multimedia messaging service via mobile phones. J Telemed Telecare. 2007; 13(4):193-196.

5. Al-Hadithy N, Gikas PD, Al-Nammari SS. Smartphones in orthopaedics. Int Orthop. 2012;36(8):1543-1547.

6. Popat R, Mohan AT, Branford OA. Current uses of smartphones and apps in orthopaedic surgery. Br J Hosp Med (Lond). 2013;74(12):672-676.
7. Orthobuzz. JBJS Mobile Technology and Social Medial Usage Study How to Become iPad-Savvy; 2014. Available from http://orthobuzz.jbjs. org/2014/09/23/how-to-become-ipad-savvy/.

8. Surgery TJoBaJ. Residents Predict Medical Apps will Play Major Role. OrthoBuzz; 2014. Available from http://orthobuzz.jbjs.org/2014/09/19/ social-media-still-slow-to-catch-on/.

9. Statista. Number of Apps Available in Leading App Stores as of July 2014; 2014. Available from: http://www.statista.com/statistics/276623/ number-of-apps-available-in-leading-app-stores/.

10. American EHR. Tablet Usage by Physicians Report - 2013; 2013. Available from: http://www.americanehr.com/research/reports/tabletreport-2013.aspx.

11. American EHR. Mobile Usage in the Medical Space Report-2013; 2013. Available from http://www.americanehr.com/research/reports/ tablet-report-2013.aspx.

12. Service NH. Health Apps Library; 2015. Available from: http://apps. nhs.uk/.

13. Andrawis J, Muzykewicz D, Franko OI. Mobile device trends in orthopaedic surgery: rapid change and future implications. Orthopedics. 2015. [In submission].

14. Peters FM, Greeff R, Goldstein N, Frey CT. Improving acetabular cup orientation in total hip arthroplasty by using smartphone technology. J Arthroplasty. 2012;27(7):1324-1330.

15. Qiao J, Liu Z, Xu L, et al. Reliability analysis of a smartphone-aided measurement method for the Cobb angle of scoliosis. J Spinal Disord Tech. 2012;25(4):E88-E92.

16. Shaw M, Adam CJ, Izatt MT, Licina P, Askin GN. Use of the iPhone for Cobb angle measurement in scoliosis. Eur Spine J. 2012;21(6): 1062-1068.

17. Shin SH, Ro du H, Lee OS, Oh JH, Kim SH. Within-day reliability of shoulder range of motion measurement with a smartphone. Man Ther. 2012;17(4):298-304.

18. Franko OI, Bray C, Newton PO. Validation of a scoliometer smartphone app to assess scoliosis. J Pediatr Orthop. 2012;32(8):e72-e75.

19. Izatt MT, Bateman GR, Adam CJ. Evaluation of the iPhone with an acrylic sleeve versus the Scoliometer for rib hump measurement in scoliosis. Scoliosis. 2012;7(1):14.

20. Ferriero G, Vercelli S, Sartorio F, et al. Reliability of a smartphonebased goniometer for knee joint goniometry. Int J Rehabil Res. 2013; 36(2):146-151.

21. Jenny JY. Measurement of the knee flexion angle with a Smartphoneapplication is precise and accurate. $J$ Arthroplasty. 2013;28(5): 784-787.

22. Kim TS, Park DD, Lee YB, et al. A study on the measurement of wrist motion range using the iPhone 4 gyroscope application. Ann Plast Surg. 2014;73(2):215-218.

23. De Maio P, White LM, Bleakney R, Menezes RJ, Theodoropoulos J. Diagnostic accuracy of an iPhone DICOM viewer for the interpretation of magnetic resonance imaging of the knee. Clin J Sport Med. 2014; 24(4):308-314.

24. Naqvi GA, Daly M, Dawood A, Kurkuri A, Kutty S. Smart consultation for musculoskeletal trauma: accuracy of using smart phones for fracture diagnosis. Surgeon. 2014;12(1):32-34.

25. Mc Laughlin P, Neill SO, Fanning N, et al. Emergency CT brain: preliminary interpretation with a tablet device: image quality and diagnostic performance of the Apple iPad. Emerg Radiol. 2012;19(2): 127-133.

26. Strayer SM, Semler MW, Kington ML, Tanabe KO. Patient attitudes toward physician use of tablet computers in the exam room. Fam Med. 2010;42(9):643-647.

27. O'Neill S, Brady RR. Clinical involvement and transparency in medical apps; not all apps are equal. Colorectal Dis. 2012;15(1):122.

28. Brady RR, Hunt AC, Visvanathan A, et al. Mobile phone technology and hospitalized patients: a cross-sectional surveillance study of bacterial colonization, and patient opinions and behaviours. Clin Microbiol Infect. 2011;17(6):830-835.

29. Rodrigues MA, Brady RR. Anaesthetists and apps: content and contamination concerns. Anaesthesia. 2011;66(12):1184-1185. 
30. Wolf JA, Moreau JF, Akilov O, et al. Diagnostic inaccuracy of smartphone applications for melanoma detection. JAMA Dermatol. 2013;149(4):422-426.

31. Haffey F, Brady RR, Maxwell S. A comparison of the reliability of smartphone apps for opioid conversion. Drug Saf. 2013;36(2):111-117.

32. Bindhim NF, Freeman B, Trevena L. Pro-smoking apps for smartphones: the latest vehicle for the tobacco industry? Tob Control. 2012;23(1):e4

33. Buijink AW, Visser BJ, Marshall L. Medical apps for smartphones: lack of evidence undermines quality and safety. Evid Based Med. 2013;18(3) 90-92.

34. McCartney M. How do we know whether medical apps work? BMJ. 2013;346:f1811

35. Administration FaD. In: Services USDoHaH, Administration FaD, editor. Mobile Medical Applications Guidance for Industry and Food and Drug Administration Staff. Silver Spring: FDA; 2013.

36. Hamilton AD, Brady RR. Medical professional involvement in smartphone 'apps' in dermatology. Br J Dermatol. 2012;167(1):220-221.
37. O'Neill S, Brady RR. Colorectal smartphone apps: opportunities and risks. Colorectal Dis. 2012;14(9):e530-e534.

38. Visvanathan A, Hamilton A, Brady RR. Smartphone apps in microbiology - is better regulation required? Clin Microbiol Infect. 2012;18(7):E218-E220.

39. Health H. Proposed NHS App Store; 2012. Available from: http:// handihealth.org/proposed-nhs-app-store/.

40. Commission FT. Mobile App Developers: Start with Security; 2013. Available from: https://www.ftc.gov/tips-advice/business-center/ guidance/mobile-app-developers-start-security.

41. Ackerman L. Mobile Health and Fitness Applications and Information Privacyl; 2013. Available from: https://www.privacyrights.org/mobilemedical-apps-privacy-consumer-report.pdf.

42. Lewis TL, Wyatt JC. mHealth and mobile medical Apps: a framework to assess risk and promote safer use. J Med Internet Res. 2014;16(9): e210.

43. AAOS. Orthopaedic Practice in the US 2012; 2013. Chicago. Available from: http://www.aaos.org/research/orthocensus/Census.asp.
Orthopedic Research and Reviews

\section{Publish your work in this journal}

Orthopedic Research and Reviews is an international, peer-reviewed, open access journal focusing on the patho-physiology of the musculoskeletal system, trauma, surgery and other corrective interventions to restore mobility and function. Advances in new technologies, materials, techniques and pharmacological agents are particularly welcome. The journal welcomes

\section{Dovepress}

original research, clinical studies, reviews \& evaluations, expert opinion and commentary, case reports and extended reports. The manuscript management system is completely online and includes a very quick and fair peer-review system, which is all easy to use. Visit http://www.dovepress. com/testimonials.php to read real quotes from published authors.

Submit your manuscript here: http://www.dovepress.com/orthopedic-research-and-reviews-journal 